\title{
Role of trans-abdominal and trans-perineal venous duplex ultrasound in cases of pelvic congestion syndrome
}

\author{
Ahmed A. Baz
}

\begin{abstract}
Background: For evaluation the role of trans-abdominal and trans-perineal venous duplex ultrasound in cases of pelvic congestion syndrome, fifty patients with pelvic congestion syndrome were included in the current research. All were evaluated by trans-abdominal and trans-perineal venous duplex.

Results: An incompetent left gonadal vein was detected in all cases with a mean diameter $( \pm S D)=7.9 \pm 1.1 \mathrm{~mm}$. The right gonadal vein was incompetent in 4 cases (8\%) with a mean diameter ( \pm SD) $5.9 \pm 0.4 \mathrm{~mm}$. A refluxing proximal internal iliac vein was detected in 3cases (6\%). Left renal vein nutcracker was present in 41 cases (82\%) while the left common iliac vein compression was present in 3 cases (6\%). Vulvoperineal varicosities were seen in all cases \{right side $=(36 \%, n=18)$, left side $=(30 \%, n=15)$, and bilateral $=(34 \%, n=17)\}$. Thigh extension of the vulvoperineal varicosities was present in $(74 \%, n=37)$. Round ligament varicosities were present in $(6 \%, n=3)$.

Conclusions: Trans-abdominal and trans-perineal venous duplex offer a simple, noninvasive, and quick technique that can help in an accurate evaluation of the ovarian vein reflux, diameters as well as the presence of vulvoperineal, and round ligament varicosities, Moreover, it is useful in the assessment of the left renal and iliac veins compression.
\end{abstract}

Keywords: Trans-abdominal, Trans-perineal, Venous-duplex, Pelvic congestion syndrome

\section{Background}

Pelvic congestion syndrome (PCS) is a condition which is characterized by chronic noncyclical (typically dull and aching) pelvic pain lasting for more than 6 months. It can affect the females during their childbearing as well as the premenopausal periods; it usually results from the venous insufficiency of either gonadal or pelvic veins. The patients can present by different manifestations including post-coital and premenstrual pain and heaviness, lower backache, in addition to the urinary symptoms like frequency as well as vulvoperineal and lower extremity varicosities. Many gynecological problems can contribute to chronic pelvic pain in females including endometriosis, adenomyosis, uterine fibroids, pelvic adhesions, uterine prolapse, and malignancies; other non-

Correspondence: ahmedbaz2012@yahoo.com

Interventional Radiology Department \& Diagnostic, Kasr Aliny School of Medicine, Cairo University, Cairo, Egypt gynecological causes include irritable bowel syndrome, urological, and psychiatric problems can also present by chronic pelvic pain [1-3].

Despite being a potentially treatable condition, it may be often misdiagnosed due to its nonspecific manifestations that could result from other gynecological and non-gynecological diseases; thus, it may be considered as a diagnosis of exclusion [1, 3].

Many predisposing factors had been associated with the condition including multiparty, obesity, and venous outflow obstruction; moreover, the hormonal status, as the decline in the estrogen levels in the premenopausal women had been described as a contributing factor that could lead to a weakness of the venous walls [3].

Diagnosis of PCS is usually based on clinical examination and the confirmatory noninvasive imaging studies including trans-abdominal duplex ultrasound, transvaginal duplex ultrasound, computed tomography $(\mathrm{CT})$ venography, and magnetic resonance (MR) venography. 
The invasive imaging studies are usually considered as the gold standard technique, and currently, they are used in the interventional purposes (selective gonadal venography with or without embolotherapy); the pelvic laparoscopy has a limited role in the diagnosis of PCS, but it has a good sensitivity for diagnosis and management of the endometriosis and the polycystic ovary disease [4].

From the etiological point of view, PCS is considered as an equivalent to the testicular varicocele in males, and it can result from venous compression like left renal vein nutcracker by the superior mesenteric artery and abdominal aorta or by the abdominal aorta and the spine in the retro-aortic left renal vein, left common iliac vein compression by the overlying right common iliac artery; a condition known as May-Thurner syndrome, or it may result from the primary valvular incompetence of the gonadal veins [1-3].

\section{Aim of the work}

The purpose of this study was to evaluate the role of trans-abdominal and trans-perineal venous duplex ultrasound in the workup of the cases presented by PCS through the evaluation of the gonadal and pelvic veins as sources of venous reflux and to detect if there is a venous compression of the left renal or the left common iliac veins.

\section{Methods}

The study enrolled 50 patients from June 2017 till March 2019 including all cases presented by pelvic congestion syndrome. The patients were referred to our department from the vascular surgery and gynecology departments after their clinical assessment.

The patients' ages ranged from 19 to 47 years with a mean age ( $35.1 \pm 7.31$ years).

\section{Inclusion criteria}

Patients diagnosed clinically as pelvic congestion syndrome and a willingness to participate in the study.

\section{Exclusion criteria}

Patients were excluded when they had other causes of chronic pelvic pain not related to the pelvic venous insufficiency (done by their gynecological assessment), and if they had iliofemoral DVT as well as the pregnant women.

The local ethics committee approved the study, and all participants gave their informed consent before being included.

\section{Imaging technique}

The patients were instructed for adequate bowel preparation through overnight fasting, adequate fluid intake, and the use of cleansing enema if necessary.
The patients were lying supine with knee flexion in order to lax the abdominal wall muscles.

Transducers: a curvilinear transducer $2-5 \mathrm{MHz}$ was used for abdominal and pelvic veins while the linear transducer 7-12 MHz was used for the superficial veins including the vulvoperineal, round ligament, and proximal thigh veins.

\section{Target veins}

External iliac veins, proximal segments of the internal iliac veins, common iliac veins, infra-renal inferior vena cava (IVC), left renal vein, parametrial and myometrial veins, gonadal veins, and the vulvoperineal veins as well as their extension (if present) to the proximal thighs (Fig. 1).

1. External iliac veins were examined by putting the probe in a transverse orientation at the inguinal crease and identification of the common femoral veins, then the external iliac veins were followed as the continuation of their relevant common femoral veins on each side then passing posteromedial to their corresponding arteries then directly dorsal to them; these veins were assessed for their patency by B-mode, color, and spectral Doppler; this should be done to exclude their obstruction, a reason for this, is that sometimes the iliac veins are thrombosed and the crossing groin venous collaterals may be misinterpreted as inguinal varicosities.

2. Internal iliac veins were examined by following the external iliac vein (with the probe in a longitudinal orientation) till the internal iliac artery is visualized then through a fine side to side sweeping movement of the probe; the proximal segment of the vein could be visualized. These veins were assessed for their patency and for venous reflux by gentle hand pressure on their anterior abdominal walls.

3. Common iliac veins were examined by following the external iliac vein till it joins the relevant internal iliac vein, and the probe movement was directed to the umbilicus, then the probe was put transversely at a level just above the umbilicus, then the common iliac veins confluence was visualized, and the crossing iliac arteries were visualized. The left common iliac vein was carefully inspected to assess for any vascular compression at this level.

4. Infra-renal inferior vena cava (IVC) was then followed by moving the probe from the level of the umbilicus in the midline to the xiphoid process; it was assessed for its patency and to exclude any compression by masses or abdominal aortic aneurysms.

5. Left renal vein was examined by visualizing the IVC just caudal to the superior mesenteric artery origin (SMA); it was assessed for its patency and if there was an extrinsic compression either by the SMA 


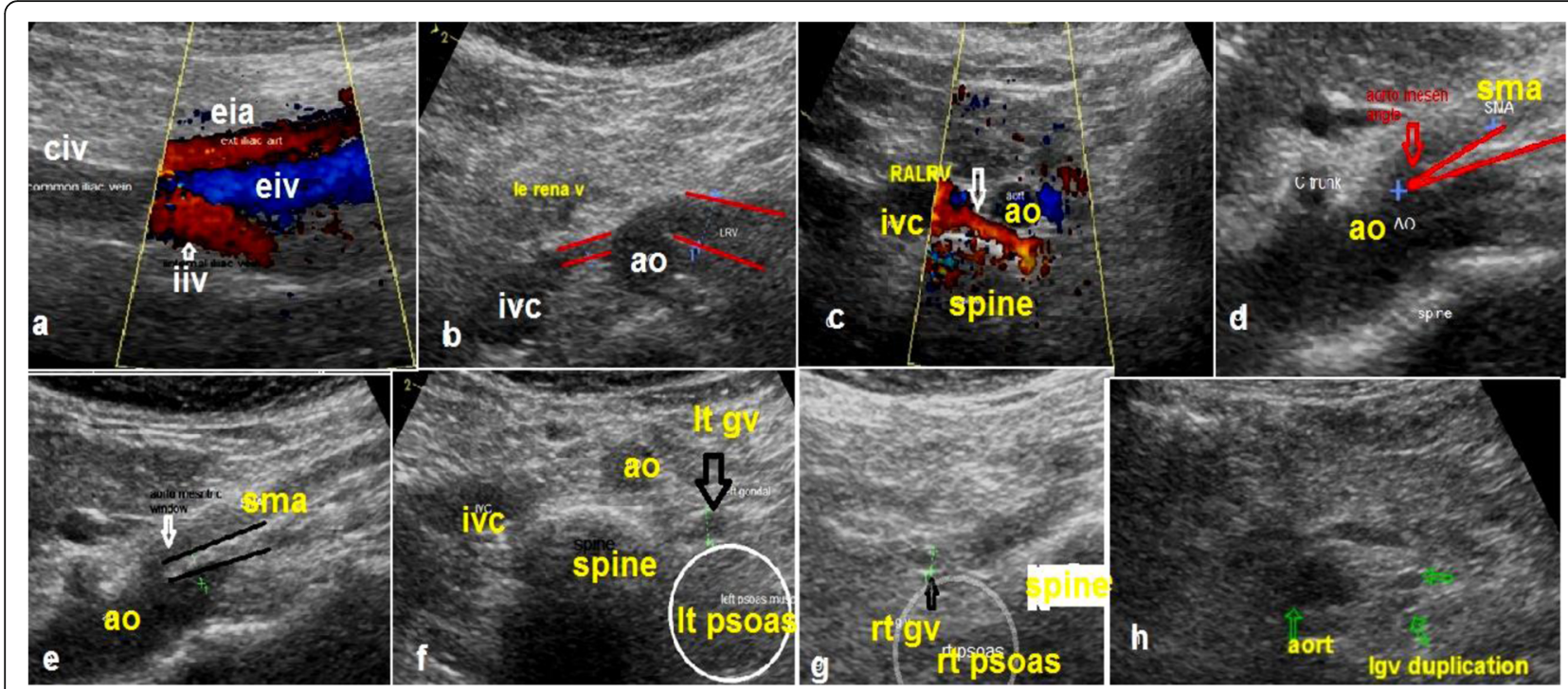

Fig. $1 \mathbf{a}-\mathbf{h}$ Trans-abdominal color Doppler and gray scale images showing a external iliac vein (eiv.) (coded in blue) just dorsal to the external iliac artery (eia) (coded in red) and the internal iliac vein (iiv) (coded in red) and the common iliac vein (civ) formed by the union of the two veins. $\mathbf{b}$ Left renal vein (between red lines) as it passed anterior to the aorta (ao) to join the IVC (ivc). c Retro-aortic left renal vein (open white arrow) (RALRV) passing posterior to the aorta (ao) anterior to the spine to join the IVC (ivc). $\mathbf{d}$ Measurement of the aorto-mesenteric angle (red lines) between aorta (ao) and superior mesenteric artery (sma). e Measurement of the aorto-mesenteric window (black lines) between aorta (ao) and superior mesenteric artery (sma). f Showing the left gonadal vein (open black arrow) (It gv) anterior to the left psoas muscle on the left side of the aorta. $\mathbf{g}$ Showing right gonadal vein (open black arrow) ( $\mathrm{rt} \mathrm{gv}$ ) anterior to the right psoas muscle on the right side of the spine. $\mathbf{h}$ Showing duplicated left gonadal vein (lgv) (open green arrows) just to the left of the aorta (aort) (open green arrow)

and aorta (anterior nutcracker) or by the aorta and the spine in cases of the retro-aortic left renal vein (RALRV); also known as posterior nutcracker.

Obstruction was assessed by narrow aortomesenteric angle (normally 22-45 degrees), narrow aorto-mesenteric distance (window) (normally $>10$ $\mathrm{mm}$ ), pre-stenotic vein dilatation with sluggish flow, and luminal reduction of the vein at the aortomesenteric window or at the retro-aortic space if there was a RALRV, in addition to, the presence of a significant rise in the velocity ratio ( $>3$ folds) and if there were any collateral veins.
6. Parametrial and transmyometrial veins were assessed by scanning the uterus in both transverse and longitudinal views; this could be done by asking the patients to fill the urinary bladder, in order to use it as an acoustic window, then the uterus was scanned and the parametrial fat planes were carefully evaluated for any dilated veins $(>4 \mathrm{~mm}$ ) and the myometrium was also scanned for any crossing dilated veins; these veins were evaluated for reflux (Fig. 2) by asking the patient to bear down or by gentle pressure of the subxiphoid area by the examiner's hand.
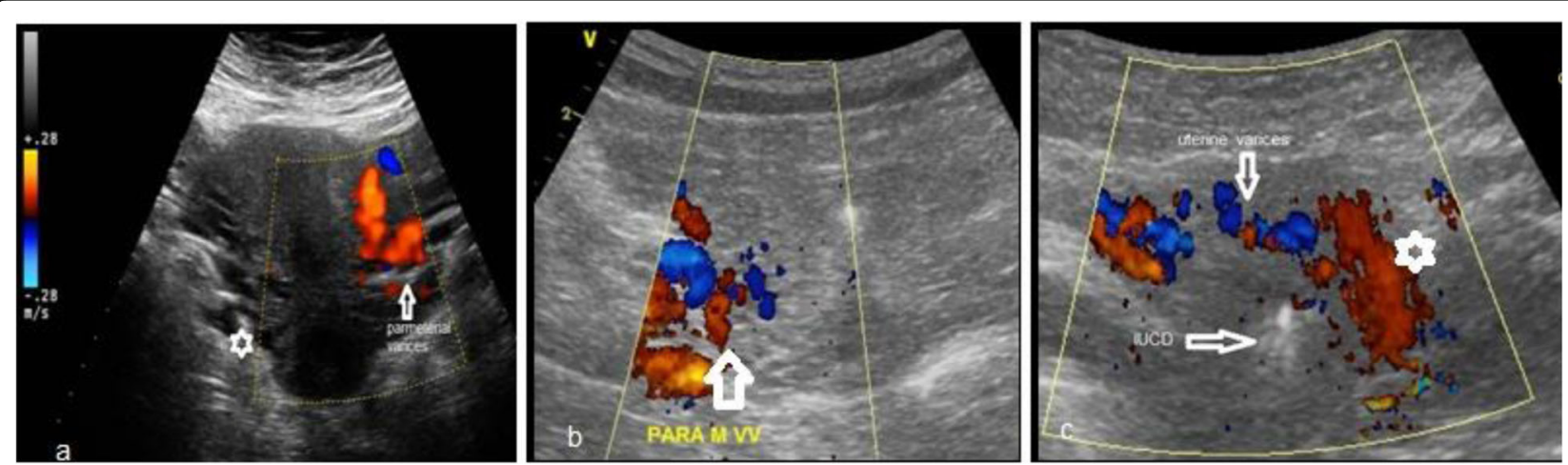

Fig. 2 a-c Trans-abdominal color Doppler images showing parametrial varices that appeared as a dilated hypoechoic cystic spaces (white asterisk) with venous reflux on raising the abdominal pressure, open white arrows in $\mathbf{a}$ and $\mathbf{b}, \mathbf{c}$ transmyometrial varices were demonstrated (vertical white arrow) and the parametrial varices (white asterisk). The horizontal arrow in (c) pointed to the echogenic IUCD 
7. Left gonadal vein was examined by putting the probe transversely to the left of the aorta at the level of the origin of the inferior mesenteric artery just anterior to the left psoas muscle (alongside pulsating left gonadal artery) then the vein was followed by moving the probe cranially to the level of the joining with the left renal vein and then the probe was moved caudally to assess the middle and distal portions of the vein, the vein was evaluated for its diameter and for the presence of significant venous reflux. A reflux could be spontaneous and continuous or occasional; Labropoulos et al. considered that spontaneous and continuous reflux in left ovarian vein as a collateral pathway circumventing the left renal vein nutcracker, while occasional reflux that occurred by raising the abdominal pressure was considered as a primary left gonadal vein reflux [1].

8. Right gonadal vein was examined by putting the probe transversely to the right of the IVC at the level of the origin of the inferior mesenteric artery just anterior to the right psoas muscle (alongside pulsating right gonadal artery) then the vein communication with the IVC could be seen at this level. The vein was evaluated for its diameter and for the presence of a reflux in the same way like the left gonadal vein.

9. Vulvoperineal veins and their thigh extension (if present) were examined after explaining the examination for the patients, then the patients were lying in the lithotomy position and by using the linear probe, the vulvoperineal regions were scanned for the presence of any dilated and refluxing veins then we continued the scanning to the proximal inner thighs to detect any communication with the thigh varicosities (Fig. 3).

10. Round ligament (inguinal) varicosities were detected at the level of the external ring, just above and lateral to the common femoral vein, they might have thigh extension to the saphenous vein or its tributaries (Fig. 3).

11. Important tips for the examination: Adequate bowel preparation may be the cornerstone of the examination, if it was not properly done, many pitfalls could result. Venous reflux could be done either by asking the patient to perform controlled Valsalva, where the patient takes a small breath and bears down with less intensity to avoid motion artifacts and displacement of the transducer and the veins, but practically, a gentle pressure by the examiner's hand just below the xiphoid process may be adequate to induce a reflux (a reflux duration more than $0.5 \mathrm{~s}$ was considered as significant reflux). Anatomical variations: many anatomical variations could be seen during the examination including duplication of the gonadal veins (that may or may not merge along the vein course) (Fig. 1), left-sided or duplicated IVC, and circum-aortic left renal vein that has an anterior limb passing anterior to the aorta and a posterior limb that passes behind the aorta; however, awareness by these anatomical variations is to be considered to avoid misinterpretation of the findings.

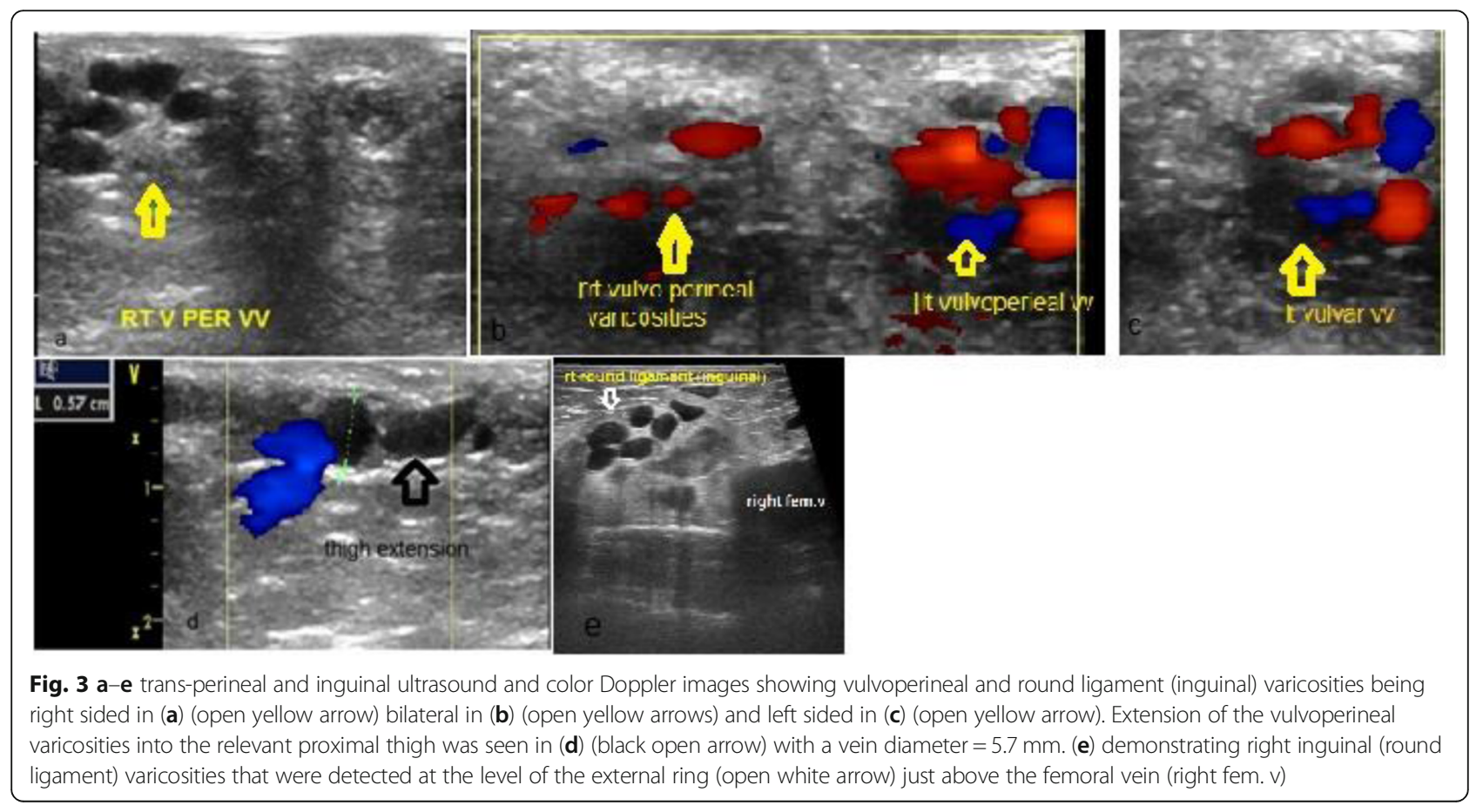


Regarding examination duration, actually, the estimated average time for full examination for a prepared non obese patient took $15-25 \mathrm{~min}$, but in the overweight and mild obese patients, the exam duration was a little bit longer and took 30-35 min; however, like any new technique, adequate practice would shorten the exam duration.

\section{Statistical methods}

Data were coded and entered using the statistical package SPSS version 25. Data were summarized using mean, standard deviation, minimum and maximum for quantitative variables and frequencies (number of cases) and relative frequencies (percentages) for categorical variables.

\section{Results}

Fifty patients with pelvic congestion syndrome were enrolled in this study. Their ages ranged from 19 to 47 years with a mean $( \pm \mathrm{SD})(35.1 \pm 7.31$ years); their primary diagnosis was established on clinical basis, then they were evaluated by trans-abdominal venous duplex for assessment of the gonadal and pelvic veins and with the trans-perineal venous duplex for assessment of the vulvoperineal and round ligament varicosities as well as their thigh extension if it was present.

In this study, the following findings were detected.

\section{Left gonadal vein}

The left gonadal vein elicited venous reflux by raising the abdominal pressure in all cases (Table 1) (Fig. 4). Spontaneous and persistent reflux was detected in only 3 cases $(6 \%)$.

The left gonadal vein dilatation was also demonstrated in all cases with a mean diameter $( \pm \mathrm{SD})=7.9 \pm 1.1 \mathrm{~mm}$ and a range $=6.5-12 \mathrm{~mm}$ (Table 2) (Fig.4).

\section{Right gonadal vein}

Right gonadal vein competence (with no pathological reflux) was demonstrated in 46 cases (92\%) (Fig. 5), and the pathological reflux was only detected in 4 cases $(8 \%)$ (Table 1) (Fig. 6), the right gonadal vein had a mean diameter $( \pm \mathrm{SD})=4.0 \pm 0.8 \mathrm{~mm}$ and a range $=2.9-6.4$ $\mathrm{mm}$ for all examined veins (Table 2 ). The refluxing right

Table 1 Demonstrating the venous reflux in the examined gonadal (gv.) and proximal internal iliac (IIV) veins and the number and percent of cases

\begin{tabular}{lll}
\hline Refluxing vein & Number & Percent (\%) \\
\hline Lt. gv. & 50 patients & 100 \\
Rt. gv. & 4 patients & 8 \\
The proximal segment of the Lt. IIV & 1 patient & 2 \\
The proximal segment of the Rt. IIV & 2 patients & 4 \\
\hline
\end{tabular}

gonadal vein had a mean diameter $( \pm$ SD) $5.9 \pm 0.4 \mathrm{~mm}$ and a range $=5.5-6.4 \mathrm{~mm}$ (Table 2).

\section{Proximal internal iliac veins}

A refluxing proximal segment of the internal iliac vein was detected in one patient $(2 \%)$ on the left side and in two patients (4\%) on the right side (Table 1) (Fig. 7).

\section{Left renal and left common iliac vein compression}

Left renal vein nutcracker was detected in 41 patients $(82 \%)$ with the anterior type (pre-aortic vein) was detected in 38 cases (76\%) (Figs. 8 and 10) while the posterior type (retro-aortic) was detected in 3 cases $(6 \%)$ (Table 3) (Fig. 1).

No nutcracker phenomenon was detected in 9 cases (18\%).

The left common iliac vein compression by the crossing right iliac artery was detected in 3 cases (6\%) (Table 3) (Fig. 9).

\section{Vulvoperineal and round ligament varicosities}

Vulvoperineal varicosities were detected in all cases (100\%) being seen on the right side in 18 cases $(36 \%)$, on the left side in 15 cases $(30 \%)$ and seen bilaterally in 17 cases (34\%) (Table 4) (Fig. 3).

Thigh extension of the vulvoperineal varicosities was detected in 37 cases (74\%) being found in 13 cases (26\%) with the right vulvoperineal varicosities, in 11 cases (22\%) with the left vulvoperineal varicosities and bilateral in 13 cases $(26 \%)$ with bilateral vulvoperineal varicosities (Table 4) (Fig. 3).

\section{Round ligament (inguinal) varicosities}

Round ligament (inguinal) varicosities were detected in 3 cases ( $6 \%$ of patients) being found on the right side in one patient (2\%) and bilateral in 2 patients (4\%) (Table 4) (Figs. 3 and 10).

\section{Discussion}

Pelvic congestion syndrome (PCS) is one of the common causes of chronic pelvic pain that predominantly affect the multiparous women, and their presentation usually occurs during their childbearing period mainly in the premenopausal age [1-3]. This was confirmed in this study, where, the patients' ages ranged from 19 to 47 years with a mean $( \pm$ SD) (35.1 \pm 7.31 years), and most of them were multiparous apart from one patient who was nulliparous. A correlation between the PCS and the decline in the ovarian activity in premenopausal women had been suggested [5].

Many diagnostic modalities were used in the diagnosis of PCS including invasive and noninvasive tests [4]. In the present study, we tried to give a simple order for the examination steps in an easy-to-perform method for 


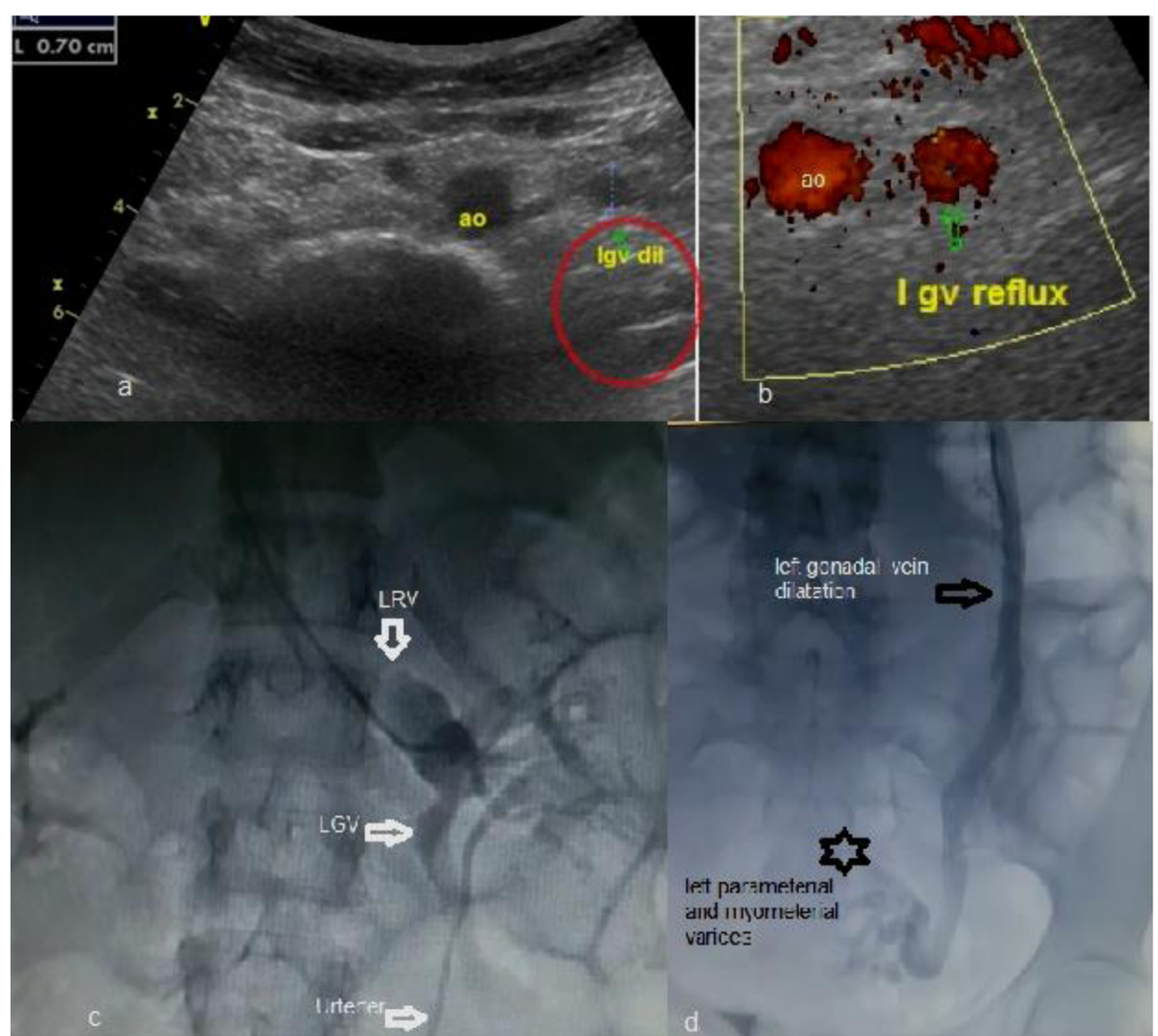

Fig. 4 a, b trans-abdominal ultrasound and color Doppler images, $\mathbf{c}$, $\mathbf{d}$ left renal and left gonadal venogram. a Showing left gonadal vein dilatation (diameter $=7 \mathrm{~mm}$ between the calibers in a) seen anterior to the psoas muscle (red circle) in (a) and to the left of the infrarenal abdominal aorta (ao). Left gonadal vein reflux (coded in red) (open green arrow) in (b) was taking the same red color of the adjacent infra-renal abdominal aorta (Ao) $\mathbf{c}$ and d left renal (LRV) (white open vertical arrow) in (c) and left gonadal vein (LGV) (white horizontal arrow) in (a) angiogram showing dilated refluxing left gonadal vein (open horizontal black arrow) in (d) down to the left parametrial and myometrial varices (black asterisk) in (d)

assessment of the PCS that can be feasible for all expert and non-expert radiologists.

CT venography (CTV) may be of great value in delineation of the precise anatomical details of the pelvic, renal, and the gonadal vein abnormalities (Fig. 10), but on the other hand, it may be of less value on the hemodynamic changes [6]; the need for contrast administration and the exposure to radiation may be considered as limitations for the use of the CTV in this issue.

Magnetic resonance imaging (MRI) is currently considered as an investigation of choice for PCS, as it provides the information about both the anatomical and flow abnormalities without exposure to ionizing radiation, unlike the CTV [7]; however, it is considered expensive when compared with the trans-abdominal venous duplex examination; moreover, MRI may not be suitable for certain patients like claustrophobic and patients with cardiac pacemakers.

Park et al. and Whiteley et al. had considered the trans-vaginal ultrasound as the gold standard for diagnosis of PCS [2, 8]; they explained this by its ability for better visualization of the parametrial veins and for detection of any other gynecological problem [8]; however, the whole course of the gonadal veins could not be assessed by the trans-vaginal route as well as the renal and the iliac veins compression; moreover, the transvaginal examination cannot be done in certain circumstances like in virgin women (we had one case).

Table 2 Demonstrating the mean diameter \pm standard deviation (SD) and the diameter range measured in millimeters (mm) for the left gonadal vein, right gonadal vein and for the right gonadal vein with pathological reflux

\begin{tabular}{lll}
\hline & Mean diameter \pm SD & Diameter range \\
\hline Left gonadal vein & $7.9 \pm 1.1 \mathrm{~mm}$ & $6.5-12 \mathrm{~mm}$ \\
Right gonadal vein & $4.0 \pm 0.8 \mathrm{~mm}$ & $2.9-6.4 \mathrm{~mm}$ \\
Right gonadal vein with pathological reflux & $5.9 \pm 0.4 \mathrm{~mm}$ & $5.5-6.4 \mathrm{~mm}$ \\
\hline
\end{tabular}




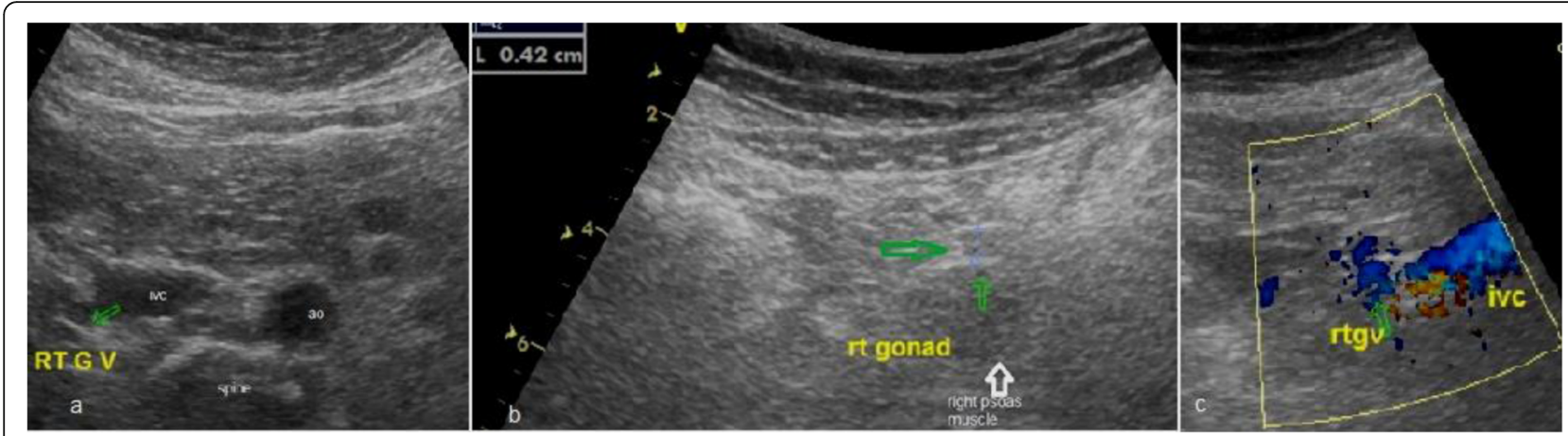

Fig. 5 a, b Trans-abdominal ultrasound and c color Doppler images showing right gonadal vein at its connection with the IVC (open green arrow in $\mathbf{a}$ ) and its measurement (diameter $=4.2 \mathrm{~mm}$ open green arrows in $\mathbf{b}$ ) seen anterior to the psoas muscle (open white arrow) in (b). Right gonadal vein competence (open green arrow) in (c) was demonstrated (coded in blue) taking the same color of the adjacent IVC (ivc)

Therefore, the trans-abdominal and the trans-perineal venous duplex scanning appeared to be a more beneficial examination, and this was greatly matching Labropoulos et al. study [1].

Being available, noninvasive, cheap, and avoiding the exposure to radiation or the use of contrast material, the trans-abdominal, and the trans-perineal venous duplex scanning had gained a great diagnostic value through providing anatomical and hemodynamic details about the examined veins in cases of PCS; nevertheless, the patients can change their positions from supine to standing and vice versa, and the examiner can do the abdominal pressure when the patients cannot perform controlled Valsalva [1].

By trans-abdominal venous duplex scanning, the left gonadal vein elicited reflux by raising the abdominal pressure either by gentle sub-xiphoid compression or by controlled Valsalva. The left gonadal vein dilatation was also demonstrable with a mean diameter $( \pm$ SD) $=7.9 \pm$ $1.1 \mathrm{~mm}$ (Table 2); this greatly matched the literature [1-3]. There was a debate about the vein diameter or the presence of reflux as a primary criterion for diagnosis of PCS; in this study, we greatly agreed with Labropoulos et al. [1] where the reflux was considered as the primary criterion; this is because all the left gonadal veins were refluxing in this study (100\%) (Table 1), but regarding the vein diameter, some authors consider gonadal vein diameter cutoffs starting above $4 \mathrm{~mm}$ as diseased veins [2]; however, the right gonadal vein was competent in 46 cases $(92 \%)$ in this study with mean diameter $( \pm \mathrm{SD})=4.0 \pm 0.8$ $\mathrm{mm}$ (Tables 1 and 2); whereas, the refluxing right gonadal vein was detected in 4 cases $(8 \%)$ with a mean diameter $( \pm$ SD) $5.9 \pm 0.4 \mathrm{~mm}$ (Tables 1 and 2); this could be explained

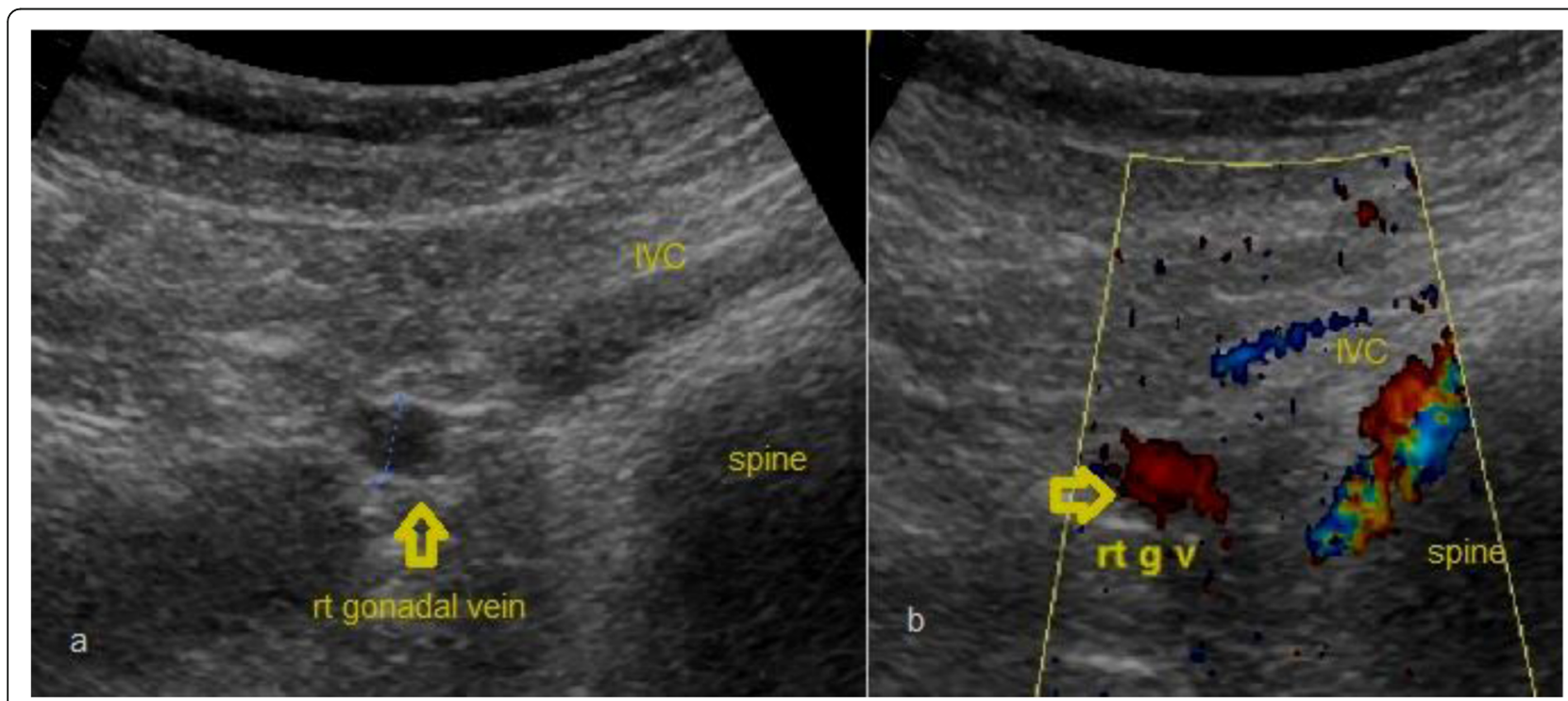

Fig. $\mathbf{6}$ a Trans-abdominal ultrasound and $\mathbf{b}$ color Doppler images showing right gonadal vein dilatation (open yellow arrow) on the right side of the IVC and the spine b) demonstrating right gonadal vein reflux (coded in red, open yellow arrow) by raising the abdominal pressure 


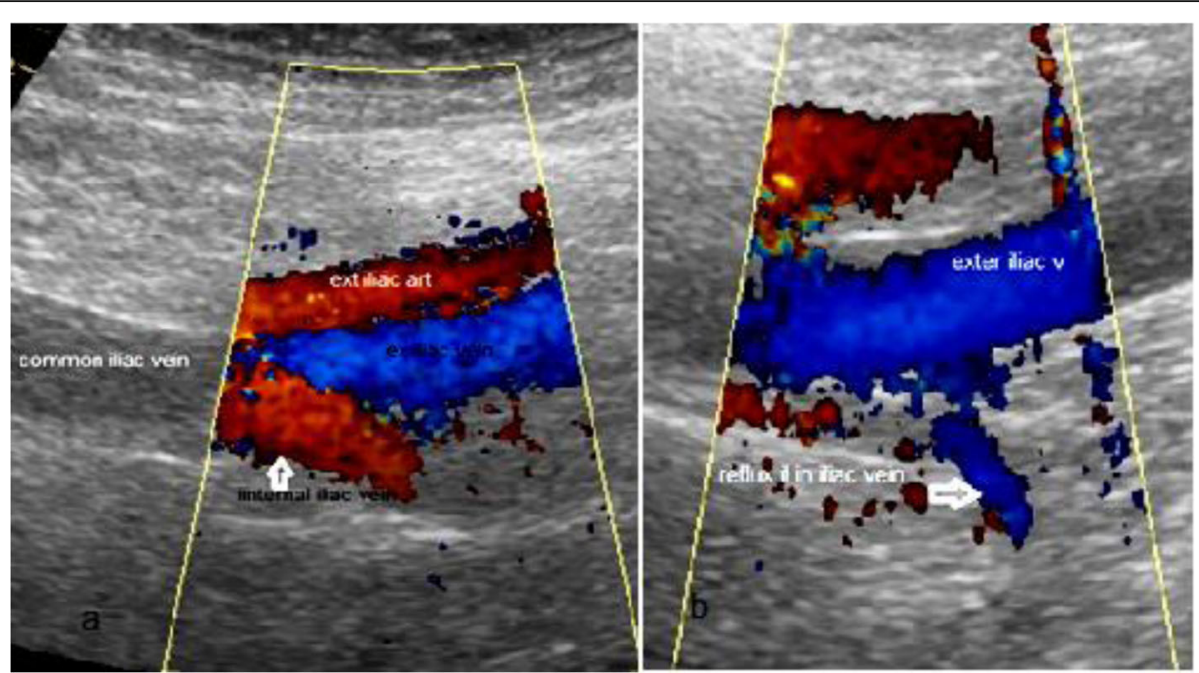

Fig. 7 a, b Trans-abdominal color Doppler images showing a competent proximal traceable portion of the internal iliac vein (coded in red) in (a) and venous reflux in the internal iliac vein (coded in blue) (open white arrow) in (b)

by the fact that the vein could be dilated to compensate for the volume overload from the contra-lateral vein reflux; in other words, a smaller but refluxing vein should be considered as a pathological vein rather than a larger but competent vein. In our experience, a venous reflux coexistent with a large vein diameter should be considered as the criteria for pelvic venous insufficiency in addition to the presence of parametrial varices.

Szaflarski et al. had recently conducted a study on a large number of patients (1042) using the CT abdomen and pelvis for evaluating the severity of the ovarian vein dilatation that was present only in 143 patients; they selected the diameter parameter as a criterion for detection of the PCS with the mean diameter for the dilated left gonadal vein $=7.5 \mathrm{~mm}$ while for the right gonadal vein $=7.2 \mathrm{~mm}$; they also found-based on quartile analysis-that the ovarian vein dilatation grading was mild $(<6 \mathrm{~mm})$ moderate $(6-8 \mathrm{~mm})$ and severe $(>8 \mathrm{~mm})$; again, in our study, we relied on the diameter and on the presence of the venous reflux (lasting

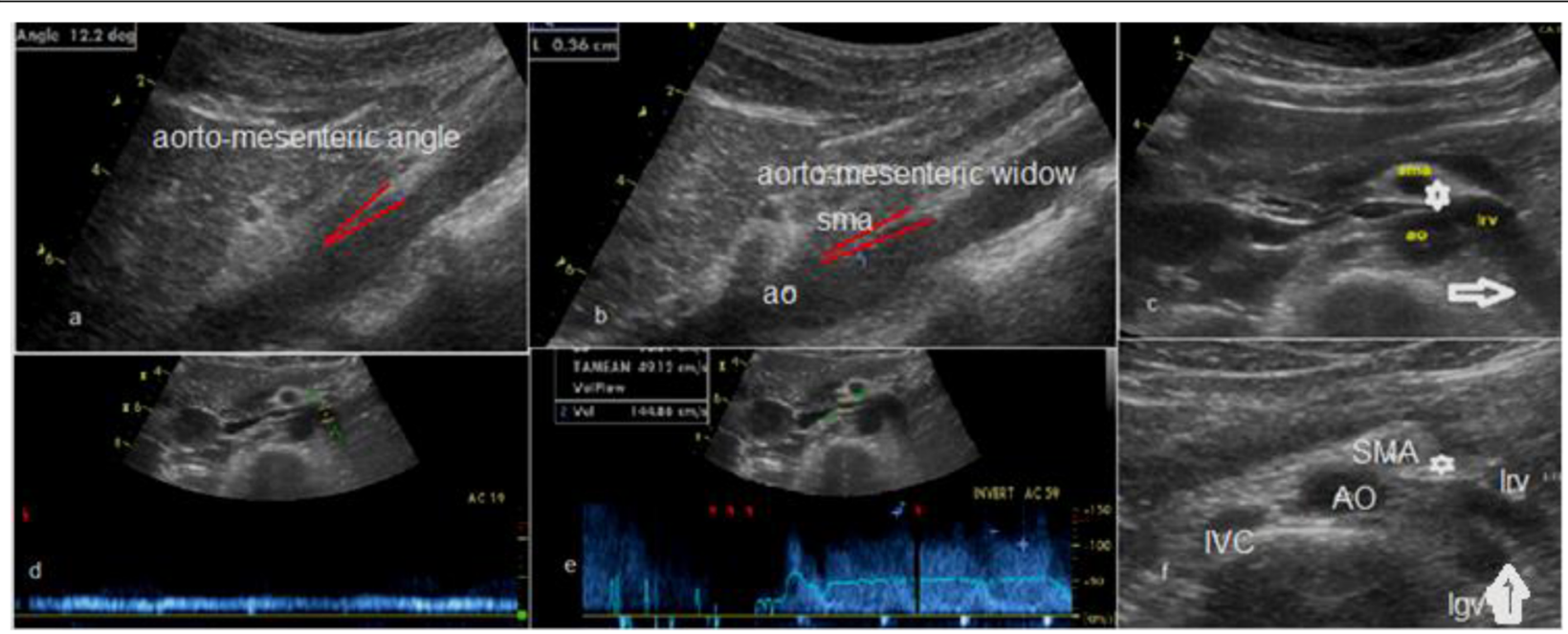

Fig. 8 a-f trans-abdominal and spectral Doppler images showing anterior nutcracker of the left renal vein evidenced by (narrow aortomesenteric angle $=12.2$ degrees and narrow aorto-mesenteric window $=3.6 \mathrm{~mm}$ between the red lines) in $(\mathbf{a}, \mathbf{b})$ significant left renal vein dilatation (open white arrow) in (c) being entrapped as it passed in the aorto-mesenteric window (white asterisk) in (c) between the aorta (ao) and superior mesenteric artery (sma); this was proven by spectral Doppler interrogation that showed significant rise in the velocity ratio in the nutcrackered segment of the vein ( $\mathbf{d} \& \mathbf{e}$ ). The left gonadal vein dilatation (open white arrow) in (f) was secondary to left renal vein nutcracker (white asterisk) in (f) as it passed in the aorto-mesenteric window 
Table 3 Demonstrating the left renal vein (LRV) nutcracker status and the left common iliac vein (LCIV) compression in the study as well as the patients' number and percent

\begin{tabular}{lll}
\hline & Number & $\%$ \\
\hline LRV with no nutcracker & 9 patients & 18 \\
LRV with nutcracker & & \\
Anterior nutcracker & 38 patients & 76 \\
Posterior nutcracker & 3 patients & 6 \\
LCIV compression (May-Thurner syndrome) & 3 patients & 6 \\
\hline
\end{tabular}

for $>0.5 \mathrm{~s}$ ), and this could explain the difference between the two studies [9].

Refluxing proximal segments of the internal iliac veins were detected in 3 patients $(6 \%)$ one of them $(2 \%)$ on the left side and two (4\%) were on the right side (Table 1); this greatly matched with Liddle and Davies, where, they consider the presence of the internal iliac vein reflux in PCS, but actually, the majority of cases with pelvic varices had ovarian vein reflux [4]. Similarly, the venous reflux was the considerable criterion rather than the vein diameter when we went for assessment of the internal iliac vein, as we noticed that the internal iliac veins may dilate secondary to refluxing ovarian vein, but their competence was still preserved.

In agreement with Zerhouni et al. and Unlu et al., in the current study, the left renal vein nutcracker phenomenon was detected in the majority of cases $(82 \%)$ being of the anterior type (pre-aortic) in (76\%) of cases and being posterior type (retro-aortic) in $(6 \%)$ of cases (Table 3); this eventually considered the left renal vein entrapment as the primary cause of left gonadal vein reflux $[10,11]$. For nutcracker phenomenon diagnosis, the aorto-mesenteric window (distance), the aortomesenteric angle, the velocity ratio in the left renal vein segments were measured as well as the diameter of the left renal vein in the aorto-mesenteric space and compared with the preceding (hilar) dilated segment $[1,12]$.

Szaflarski et al. had described the presence of nutcracker only in $14.4 \%$ of their patients, but this could be explained by the different sample sizes where they included 143 patients in their study [9].

The left common iliac vein compression by the crossing right iliac artery was detected in 3 of our cases $(6 \%)$ (Table 3) by using the trans-abdominal ultrasound examination [1]; however, the internal iliac veins proximal segments in these cases were competent; this may emphasize that the pelvic venous insufficiency is attributed mainly to the gonadal vein reflux rather than the iliac vein reflux; this was concordant with Rastogi et al. study, where they considered the two conditions being unrelated [13].

Craig and Hobbs and Hobbs had declared a strong association between PCS and the varicosities that were detected in the vulva as well as their extensions in the thighs and buttocks especially, if the varicosities had atypical distribution in the lower limbs, if there was a recurrence with no obvious cause or in those with primarily failed surgical treatment [14, 15].

Jung et al.'s study considered the vulvoperineal and the round ligament varicosities as parts from the PCS; they also found that about $85-90 \%$ of the unusual causes of the lower limb varicosities were arising primarily from the vulvoperineal varicosities then from the round ligament varicosities [16]. In our study, we searched for the vulvoperineal and the round ligament varicosities in all of our cases, and we found that vulvoperineal varicosities were present in all cases of PCS $(100 \%$ of cases, $n=50)$, and to lesser extent, round ligament varicosities were present ( $6 \%$ of cases, $n=3$ ), and the thigh extension of the vulvoperineal varicosities was found in (74\% of cases) and in all cases (3\%) with round ligament varicosities (Table 4); this high proportion of thigh extension of the

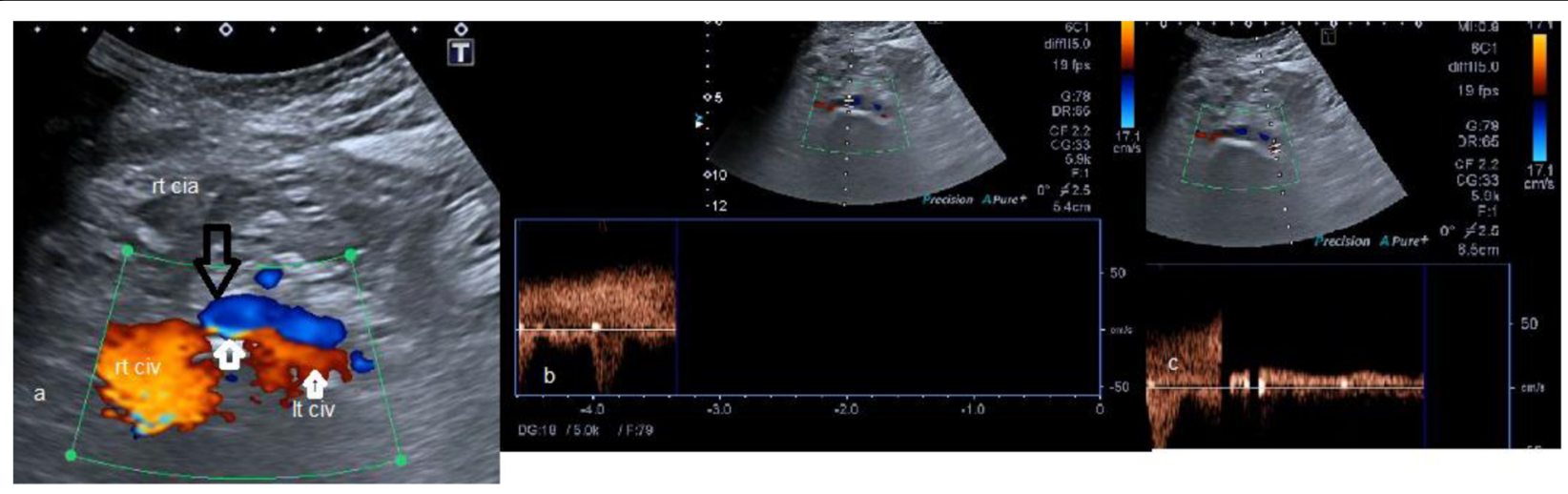

Fig. 9 a-c trans-abdominal color and spectral Doppler images showing significant compression of the left common iliac vein (It.civ.) (Open white arrows in a) by the right common iliac artery ( $r$. cia.) with significant rise in the velocity ratio that was measured in the $\mathbf{b}$ compressed segment as compared with the preceding $\mathbf{c}$ uncompressed segment 
Table 4 Demonstrating the presence of the vulvoperineal varicosities, side of affection, thigh extension and the number and percent of the affected cases as well as the round ligament (inguinal) varicosities

\begin{tabular}{lll}
\hline Vulvoperineal varicosities & Number & $\%$ \\
\hline Right side & 18 patients & 36 \\
Thigh extension & 13 patients & 26 \\
No thigh extension & 5 patients & 10 \\
Left side & 15 patients & 30 \\
Thigh extension & 11 patients & 22 \\
No thigh extension & 4 patients & 8 \\
Bilateral & 17 patients & 34 \\
Thigh extension & 13 patients & 26 \\
No thigh extension & 4 patients & 8 \\
Round ligament (inguinal) varicosities & 3 patients & 6 \\
Right side & 1 patient & 2 \\
Bilateral & 2 patients & 4 \\
\hline
\end{tabular}

vulvoperineal varicosities was rationale as all patients in this study were referred mainly from the vascular surgery department with atypical limb varicosities, and the patients' clinical diagnosis was PCS.

Recently, a correlation between the ovarian varices and infertility had been found in some patients, and the effect of the gonadal vein embolization on the subsequent conception was studied by Liu et al., and they reported the safety and the efficacy of the technique for the women trying to have conception [17].

Unlike Niclot et al. and Iupatov et al. studies, we excluded pregnant women from our study and those who had an ilio-femoral DVT (post thrombotic PCS) as we were focused to establish the technique in the patients who had primary venous insufficiency related to the gonadal or the iliac veins $[18,19]$.

In agreement with the literature, PCS is not uncommon entity that often misdiagnosed or undiagnosed clinically and radiologically, thus we recommended that one should consider the diagnosis PCS in the diagnostic list when evaluating the female pelvis for chronic pelvic pain and in cases of atypical lower limb varicosities, especially, in limbs where the varicosities assemble to the proximal inner thighs and to the perineum.

Several limitations were met in this work; the first one was regarding the technique of examination, being an operator dependent technique that needed experience and repeated practice for reducing the duration of the examination; the second one, was that the trans-abdominal studies depends greatly on the good bowel preparation and the body habitus; however, this was overcome by an overnight fasting, changing the patient position during the examination, and cleansing enemas when needed; the third one was the lack of control (healthy) group for detection of the cutoffs for normal gonadal veins diameters; however, this was described by other studies; the last one was the absence of a gold standard comparative imaging study (like selective gonadal venography, CTV or MRV); this was attributable to that many patients did not go for further imaging work up especially if they had no intention of doing interventional procedures.

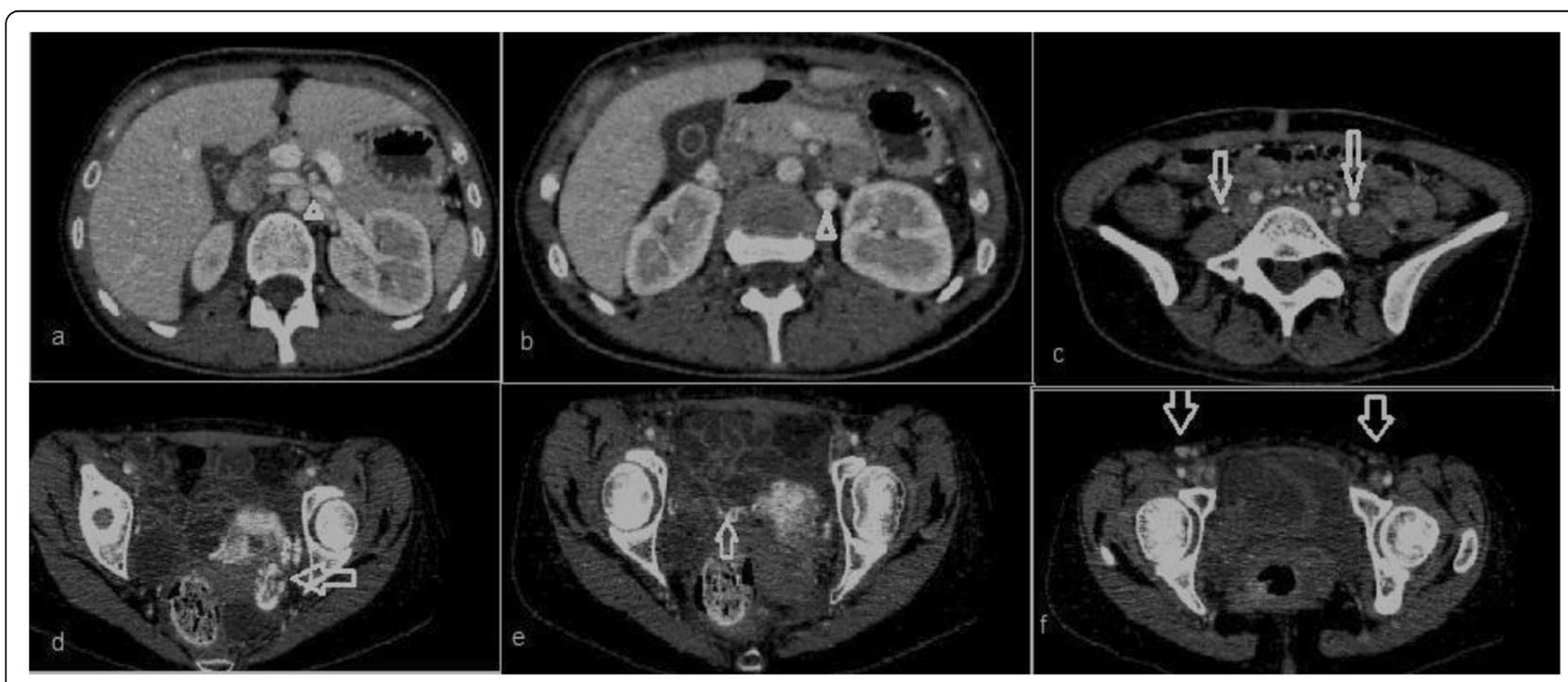

Fig. 10 a CTV axial images showing nutcracker of the pre-aortic left renal vein at $\mathbf{b}$ the aorto-mesenteric window (arrow head) showing $\mathbf{c}$ left gonadal vein dilatation (arrow head) showing the difference in caliber between the right and $\mathbf{d}$ left gonadal veins (open white arrows) showing $\mathbf{e}$ left parametrial varices (open white side arrow) showing $\mathbf{f}$ crossing parametrial varices (open white arrow) superficial inguinal varicosities seen more on the right side (open white arrows) 


\section{Conclusion}

Pelvic congestion syndrome is one of the common causes of chronic pelvic pain that is often misdiagnosed but still having a potential curability; its diagnosis is made by exclusion of other gynecological and nongynecological causes. Trans-abdominal and trans-perineal venous duplex ultrasound offers a safe, a simple, noninvasive, and quick technique that can help in the dynamic assessment of pelvic venous insufficiency through evaluation of the ovarian vein incompetence, diameter, and the presence of vulvoperineal and round ligament varicosities, Moreover, it is useful in the assessment of the left renal vein and the iliac veins being an outflow for the pelvic venous drainage.

\section{Abbreviations}

CTV: Computed tomography venography; MRV: Magnetic resonance venography; PCS: Pelvic congestion syndrome

\section{Acknowledgements}

Not applicable.

\section{Author's contributions}

$A A B$, the corresponding author, had contributed by doing ultrasound examinations and the interpretation of the corresponding correlative image studies if available in the research work. He also had introduced the idea of the current study and helped in the image selection and in the editing of the manuscript and reference collection. The author of this paper have read and approved the final version submitted.

\section{Funding}

The author had no fund for this research and had no competing interests.

\section{Availability of data and materials}

All data are available on a software system owned by each of the authors and the corresponding author has the authority to respond if there is any query.

\section{Ethics approval and consent to participate}

The protocol was reviewed and approved by the local ethics committee of the radiology department, Kasr Aliny hospital, Cairo University.

The reference number was not applicable.

All patients had given their written consents to participate in this work.

\section{Consent for publication}

All patients had given their written consents for publication of this work.

\section{Competing interests}

The authors declare that they have no competing interests.

Received: 27 November 2019 Accepted: 2 December 2019

Published online: 16 December 2019

\section{References}

1. Labropoulos N, Jasinski P, Adrahtas D, Gasparis A, Meissner M (2016) A standardized ultrasound approach to pelvic congestion syndrome. Phlebology 32(9):608-619

2. Park S, Lim J, Ko Y, Lee D, Yoon Y, Oh J, Lee H, Huh C (2004) Diagnosis of pelvic congestion syndrome using transabdominal and transvaginal sonography. Am J Roentgenol 182(3):683-688

3. Ignacio E, Dua R, Sarin S, Harper A, Yim D, Mathur V, Venbrux A (2008) Pelvic congestion syndrome: diagnosis and treatment. Semin Interv Radiol 25(04):361-368

4. Liddle A, Davies A (2007) Pelvic congestion syndrome: chronic pelvic pain caused by ovarian and internal iliac varices. Phlebology 22(3):100-104
5. Adams J, Reginald P, Franks S, Wadsworth J, Beard R (1990) Uterine size and endometrial thickness and the significance of cystic ovaries in women with pelvic pain due to congestion. BJOG 97(7):583-587

6. Desimpelaere J, Seynaeve P, Hagers Y, Appel B, Mortelmans L (1999) Pelvic congestion syndrome: demonstration and diagnosis by helical $\mathrm{CT}$. Abdom Imaging 24(1):100-102

7. Knuttinen M, Xie K, Jani A, Palumbo A, Carrillo T, Mar W (2015) Pelvic venous insufficiency: imaging diagnosis, treatment approaches, and therapeutic issues. Am J Roentgenol 204(2):448-458

8. Whiteley M, Dos Santos S, Harrison C, Holdstock J, Lopez A (2014) Transvaginal duplex ultrasonography appears to be the gold standard investigation for the hemodynamic evaluation of pelvic venous reflux in the ovarian and internal iliac veins in women. Phlebology 30(10):706-713

9. Szaflarski D, Sosner E, French T, Sayegh S, Lamba R, Katz D, Hoffmann J (2018) Evaluating the frequency and severity of ovarian venous congestion on adult computed tomography. Abdom Radiol 44(1):259-263

10. Zerhouni E, Siegelman S, Walsh P, White R (1980) Elevated pressure in the left renal vein in patients with varicocele: preliminary observations. J Urol 123(4):512-513

11. Unlu M, Orguc S, Serter S, Pekindil G, Pabuscu Y (2007) Anatomic and hemodynamic evaluation of renal venous flow in varicocele formation using color Doppler sonography with emphasis on renal vein entrapment syndrome. Scand J Urol Nephrol 41(1):42-46

12. Kurklinsky A, Rooke T (2010) Nutcracker phenomenon and nutcracker syndrome. Mayo Clin Proc 85(6):552-559

13. Rastogi N, Kabutey N, Kim D (2012) Incapacitating pelvic congestion syndrome in a patient with a history of May-Thurner syndrome and left ovarian vein embolization. Ann Vasc Surg 26(5):732.e7-732.e11

14. Craig O, Hobbs J (1975) Vulval phlebography in the pelvic congestion syndrome. Clin Radiol 26:517-525

15. Hobbs J (2005) Varicose veins arising from the pelvis due to ovarian vein incompetence. Int J Clin Pract 59(10):1195-1203

16. Jung S, Lee W, Chung J, Jae H, Park E, Jin K, Shin C, Park J (2009) Unusual causes of varicose veins in the lower extremities: $C T$ venographic and Doppler US findings. RadioGraphics 29(2):525-536

17. Liu J, Han L, Han X (2019) The effect of a subsequent pregnancy after ovarian vein embolization in patients with infertility caused by pelvic congestion syndrome. Acad Radiol. https://doi.org/10.1016/j.acra.2018.12.024

18. Niclot J, Stansal A, Di Primio M, Angelopoulos G, Yannoutsos A, Lazareth I, Priollet $P$ (2018) A post-thrombotic pelvic congestion syndrome. Journal de Médecine Vasculaire 43(5):310-315

19. Iu lupatov $E$, Ignat'ev IM, Fomina EE (2018) Ultrasonographic examination of major veins of lower limbs and pelvic veins in pregnant women. Angiol Vasc Surg 24(3):70-75

\section{Publisher's Note}

Springer Nature remains neutral with regard to jurisdictional claims in published maps and institutional affiliations.

\section{Submit your manuscript to a SpringerOpen ${ }^{\circ}$ journal and benefit from:}

- Convenient online submission

- Rigorous peer review

- Open access: articles freely available online

- High visibility within the field

- Retaining the copyright to your article

Submit your next manuscript at $>$ springeropen.com 